\title{
Erratum: Lepton flavor changing Higgs decays in the littlest Higgs model with T-parity
}

\author{
Francisco del Aguila, ${ }^{a}$ Lluis Ametller, ${ }^{b}$ Jose Ignacio Illana, ${ }^{a}$ Jose Santiago, ${ }^{a}$ \\ Pere Talavera $^{c}$ and Roberto Vega-Morales ${ }^{a}$ \\ ${ }^{a}$ CAFPE and Departamento de Física Teórica y del Cosmos, Universidad de Granada, \\ E-18071 Granada, Spain \\ ${ }^{b}$ Departament de Física, Universitat Politècnica de Catalunya, \\ E-08034 Barcelona, Spain \\ ${ }^{c}$ Institut de Ciencies del Cosmos, Universitat de Barcelona (IEEC-UB), \\ Marti i Franques 1, Barcelona 08028, Spain \\ E-mail: faguila@ugr.es, lluis.ametller@upc.edu, jillana@ugr.es, \\ jsantiago@ugr.es, ptalavera@iese.edu, rvegamorales@ugr.es
}

ERratum TO: JHEP08(2017)028

ArXiv EPrint: 1705.08827

1. The sentence in between eqs. (2.16) and (2.17) should read:

$\Psi_{R}$ transforms under T-parity:

2. Replace the sentence following eq. (2.23) by:

An explicit realization of this extra scalar factor is obtained identifying $\chi$ with $\left(\Sigma_{33}^{\dagger}\right)^{-\frac{1}{4}}$, and $\tilde{\chi}$ with $\left(\Sigma_{33}\right)^{-\frac{1}{4}}$, which have the correct $Y_{1,2}$ charges, $\left(Y_{1}^{\chi}, Y_{2}^{\chi}\right)=$ $\left(-\frac{1}{10}, \frac{1}{10}\right)$ and $\left(Y_{1}^{\tilde{\chi}}, Y_{2}^{\tilde{\chi}}\right)=\left(\frac{1}{10},-\frac{1}{10}\right)$, and T-transformation properties [19].

3. Remove the factor $\sqrt{2}$ from eq. (2.37). It should read:

$$
\lambda_{i j} v=\left(V_{L}^{\ell}\right)_{i k} m_{\ell_{k}}\left(V_{R}^{\ell \dagger}\right)_{k j} .
$$

4. There is a subindex misprinted in eq. (2.40). It should be:

$$
V \equiv V_{L}^{H \dagger} V_{L}^{\ell}, \quad W \equiv \tilde{V}_{L}^{T} V_{R}^{H}
$$

5. Although the codes, calculations and conclusions of the paper are correct, tables 2,4 , 5 and 7 contain some typos in the published version. The revised tables are collected below. 


\begin{tabular}{|c|c|c|}
\hline$[\mathrm{SFF}]$ & $c_{L}$ & $c_{R}$ \\
\hline$h \overline{\ell_{i}} \ell_{j}$ & $-\delta_{i j} \frac{m_{\ell_{j}}}{v}\left(1-\frac{v^{2}}{6 f^{2}}\right)$ & $-\delta_{i j} \frac{m_{\ell_{j}}}{v}\left(1-\frac{v^{2}}{6 f^{2}}\right)$ \\
$h \overline{\nu_{H i}} \nu_{H j}$ & $\frac{m_{\ell_{H i}}}{v} \delta_{i j} \frac{v^{2}}{4 f^{2}}$ & $\frac{m_{\ell_{H i}}}{v} \delta_{i j} \frac{v^{2}}{4 f^{2}}$ \\
$h \overline{\ell_{H i}} \ell_{H j}$ & 0 \\
$h \overline{\nu_{H i}} \tilde{\nu}_{j}^{c}$ & 0 & $\frac{m_{\ell_{H i}}}{v} W_{i j}^{\dagger} \frac{v^{2}}{4 f^{2}}$ \\
$\Phi^{0} \overline{\ell_{H i}} \ell_{j}$ & 0 & $V_{i j} \frac{m_{\ell_{j}}}{\sqrt{2} f}\left(1+\frac{v^{2}}{4 f^{2}}\right)$ \\
$\Phi^{P} \overline{\ell_{H i}} \ell_{j}$ & $i V_{i j} \frac{m_{\ell_{j}}}{\sqrt{2} f}\left(1+\frac{v^{2}}{4 f^{2}}\right)$ \\
$\Phi^{+} \overline{\nu_{H i}} \ell_{j}$ & 0 & $V_{i j} \frac{m_{\ell_{j}}}{\sqrt{2} f}\left(1-\frac{v^{2}}{8 f^{2}}\right)$ \\
$\Phi^{+} \overline{\tilde{\nu}_{i}^{c}} \ell_{j}$ & $\frac{m_{\ell_{H i}}}{\sqrt{2} f} V_{i j} \frac{v^{2}}{8 f^{2}}$ & 0 \\
$\Phi^{++} \overline{\tilde{\ell}_{i}^{c}} \ell_{j}$ & $W_{i k} \frac{m_{\ell_{H k}}}{\sqrt{2} f} V_{k j}$ & 0 \\
$\eta \overline{\ell_{H i}} \ell_{j}$ & $-W_{i k} \frac{m_{\ell_{H k}}}{f} V_{k j}$ & $m_{\ell_{H i}} V_{i j}\left[1-\left(\frac{5}{8}+x_{H} t_{W}\right) \frac{v^{2}}{f^{2}}\right]$ \\
$\omega^{0} \overline{\ell_{H i}} \ell_{j}$ & $i \frac{m_{\ell_{H i}}}{2 f} V_{i j}\left[1-\left(\frac{1}{8}-\frac{x_{H}}{t_{W}}\right) \frac{v^{2}}{f^{2}}\right]$ & $-i V_{i j} \frac{m_{\ell_{j}}}{2 \sqrt{5} f}\left[1-\left(\frac{5}{8}+x_{H} t_{W}\right) \frac{m_{\ell_{j}}}{2 f}\left[1-\left(\frac{1}{8}-\frac{v_{H}}{t_{W}}\right) \frac{v^{2}}{f^{2}}\right]\right.$ \\
$\omega^{+} \overline{\nu_{H i}} \ell_{j}$ & $-i \frac{m_{\ell_{H i}}}{\sqrt{2} f} V_{i j}$ & $i V_{i j} \frac{m_{\ell_{j}}}{\sqrt{2} f}\left(1+\frac{v^{2}}{8 f^{2}}\right)$ \\
$\omega^{+} \overline{\tilde{\nu}_{i}^{c}} \ell_{j}$ & $i W_{i k} \frac{m_{\ell_{H k}}}{\sqrt{2} f} V_{k j} \frac{v^{2}}{8 f^{2}}$ & 0 \\
\hline
\end{tabular}

Table 2. Scalar-Fermion-Fermion couplings at $\mathcal{O}\left(\frac{v^{2}}{f^{2}}\right)$. We use $t_{W}=\tan \theta_{W}=\frac{s_{W}}{c_{W}}$. (The coupling $\Phi^{++} \overline{\tilde{\ell}_{i}^{c}} \ell_{j}$ does not enter in the calculation of $h \rightarrow \bar{\ell} \ell^{\prime}$, but it does in $Z, \gamma \rightarrow \bar{\ell} \ell^{\prime}$ in order to cancel other contributions.)

\begin{tabular}{|c|c|c|}
\hline$[\mathrm{SSFF}]$ & $f_{L}$ & $f_{R}$ \\
\hline$h \Phi^{0} \overline{\ell_{H i}} \ell_{j}$ & 0 & $V_{i j} \frac{m_{\ell_{j}}}{\sqrt{2} v f}\left(1+\frac{5 v^{2}}{12 f^{2}}\right)$ \\
$h \Phi^{P} \overline{\ell_{H i}} \ell_{j}$ & 0 & $i V_{i j} \frac{m_{\ell_{j}}}{\sqrt{2} v f}\left(1+\frac{5 v^{2}}{12 f^{2}}\right)$ \\
$h \Phi^{+} \overline{\nu_{H i}} \ell_{j}$ & $-\frac{m_{\ell_{H i}}}{\sqrt{2} v f} V_{i j} \frac{v^{2}}{12 f^{2}}$ & $V_{i j} \frac{m_{\ell_{j}}}{\sqrt{2} v f}\left(1-\frac{7 v^{2}}{24 f^{2}}\right)$ \\
$h \Phi^{+} \overline{\tilde{\nu}_{i}^{c}} \ell_{j}$ & $-W_{i k} \frac{m_{\ell_{H k}}}{\sqrt{2} v f} V_{k j} \frac{v^{2}}{12 f^{2}}$ & 0 \\
$h \eta \overline{\ell_{H i}} \ell_{j}$ & 0 & $-i V_{i j} \frac{m_{\ell_{j}}}{2 \sqrt{5} v f}\left[1+\left(\frac{7}{8}-x_{H} \frac{s_{W}}{c_{W}}\right) \frac{v^{2}}{f^{2}}\right]$ \\
$h \omega^{0} \overline{\ell_{H i}} \ell_{j}$ & 0 & $-i V_{i j} \frac{m_{\ell_{j}}}{2 v f}\left[1-\left(\frac{5}{8}-x_{H} \frac{c_{W}}{s_{W}}\right) \frac{v^{2}}{f^{2}}\right]$ \\
$h \omega^{+} \overline{\nu_{H i}} \ell_{j}$ & $i \frac{m_{\ell_{H i}}}{\sqrt{2} v f} V_{i j} \frac{v^{2}}{12 f^{2}}$ & $i V_{i j} \frac{m_{\ell_{j}}}{\sqrt{2} v f}\left(1-\frac{v^{2}}{24 f^{2}}\right)$ \\
$h \omega^{+} \overline{\tilde{\nu}_{i}^{c}} \ell_{j}$ & $i W_{i k} \frac{m_{\ell_{H k}}}{\sqrt{2} v f} V_{k j} \frac{v^{2}}{12 f^{2}}$ & 0 \\
\hline
\end{tabular}

Table 4. Scalar-Scalar-Fermion-Fermion couplings at $\mathcal{O}\left(\frac{v^{2}}{f^{2}}\right)$. The coupling $h \phi^{++} \overline{\tilde{\ell}_{i}^{c}} \ell_{j}$ does vanish. 


\begin{tabular}{|c|c|}
\hline$\left[\mathrm{S}\left(p_{1}\right) \mathrm{S}\left(p_{2}\right) \mathrm{V}_{\mu}\right]$ & $G$ \\
\hline$h \Phi^{+} W_{H}^{-}$ & $g \frac{v}{4 f}$ \\
$h \eta A_{H}^{-}$ & $i \sqrt{5} g^{\prime} \frac{v}{4 f}$ \\
$h \omega^{0} Z_{H}^{-}$ & $-i g \frac{v}{4 f}$ \\
$h \omega^{+} W_{H}^{-}$ & $-i g \frac{v}{4 f}$ \\
\hline
\end{tabular}

\begin{tabular}{|c|c|}
\hline$\left[\mathrm{SS}\left(p_{1}\right) \mathrm{S}\left(p_{2}\right)\right]$ & $J$ \\
\hline$h \Phi^{0} \Phi^{0}$ & $\frac{v}{6 f^{2}}$ \\
$h \Phi^{P} \Phi^{P}$ & $\frac{v}{6 f^{2}}$ \\
$h \Phi^{P} \eta$ & $-\sqrt{\frac{5}{2}} \frac{v}{6 f^{2}}$ \\
$h \Phi^{P} \omega^{0}$ & $\frac{1}{\sqrt{2}} \frac{v}{6 f^{2}}$ \\
$h \Phi^{+} \Phi^{-}$ & $\frac{v}{12 f^{2}}$ \\
$h \Phi^{+} \omega^{-}$ & $i \frac{v}{12 f^{2}}$ \\
$h \eta \eta$ & $\frac{5 v}{12 f^{2}}$ \\
$h \eta \omega^{0}$ & $-\frac{\sqrt{5} v}{12 f^{2}}$ \\
$h \omega^{0} \omega^{0}$ & $\frac{v}{12 f^{2}}$ \\
$h \omega^{+} \omega^{-}$ & $\frac{v}{12 f^{2}}$ \\
\hline
\end{tabular}

Table 5. Scalar-Scalar-Vector and Scalar-Scalar-Scalar couplings at $\mathcal{O}\left(\frac{v^{2}}{f^{2}}\right)$. Other combinations also involving $h$ vanish.

\begin{tabular}{|r|cccccccc|c|}
\hline$C_{\mathrm{UV}}^{\left(\frac{v^{2}}{f^{2}}\right)}$ & I & II & III & IV & V+VI & VII + VIII & IX+X & XI + XII & Sum \\
\hline$W_{H}, \nu_{H}$ & 0 & 0 & - & - & - & - & - & - & 0 \\
$W_{H}, \omega, \nu_{H}$ & - & - & - & - & 0 & - & - & - & 0 \\
$\omega, \nu_{H}$ & - & - & $\frac{1}{4}$ & $-\frac{1}{8}$ & - & - & $-\frac{1}{6}$ & $\frac{5}{24}$ & $\frac{1}{6}$ \\
\hline$Z_{H}, \ell_{H}$ & $\bullet$ & 0 & - & - & - & - & - & - & 0 \\
$Z_{H}, \omega^{0}, \ell_{H}$ & - & - & - & - & 0 & - & - & - & 0 \\
$\omega^{0}, \ell_{H}$ & - & - & - & $-\frac{1}{16}$ & - & - & $-\frac{5}{24}+x_{H} \frac{c_{W}}{s_{W}}$ & $\frac{3}{8}-x_{H} \frac{c_{W}}{s_{W}}$ & $\frac{5}{48}$ \\
\hline$A_{H} \ell_{H}$ & $\bullet$ & 0 & - & - & - & - & - & - & 0 \\
$A_{H}, \eta, \ell_{H}$ & - & - & - & - & 0 & - & - & - & 0 \\
$\eta, \ell_{H}$ & - & - & - & $-\frac{1}{16}$ & - & - & $-\frac{17}{120}-x_{H} \frac{s}{5 c_{W}}$ & $-\frac{1}{40}+x_{H} \frac{s}{5 c_{W}}$ & $-\frac{11}{48}$ \\
\hline$Z_{H}, A_{H}, \ell_{H}$ & - & 0 & - & - & - & - & - & - & 0 \\
$\omega^{0}, \eta, \ell_{H}$ & - & - & - & $\frac{1}{8}$ & - & - & - & - & $\frac{1}{8}$ \\
\hline$W_{H}, \Phi, \nu_{H}$ & - & - & - & - & 0 & - & - & - & 0 \\
$\Phi, \nu_{H}$ & - & - & - & $\bullet$ & - & - & $-\frac{1}{8}$ & $\frac{1}{24}$ & $-\frac{1}{12}$ \\
\hline$\omega, \Phi, \nu_{H}$ & - & - & - & $\frac{1}{6}$ & - & - & - & - & $\frac{1}{6}$ \\
\hline$\omega^{0}, \Phi^{P}, \ell_{H}$ & - & - & - & $\frac{1}{24}$ & - & - & - & - & $\frac{1}{24}$ \\
\hline$\eta, \Phi^{P}, \ell_{H}$ & - & - & - & $-\frac{1}{24}$ & - & - & - & - & $-\frac{1}{24}$ \\
\hline$\Phi, \tilde{\nu}^{c}$ & - & - & $-\frac{1}{4}$ & $\frac{1}{24}$ & - & - & - & $-\frac{1}{24}$ & $-\frac{1}{4}$ \\
\hline Total & 0 & 0 & 0 & $\frac{1}{12}$ & 0 & - & $-\frac{47}{120}$ & $\frac{37}{120}$ & 0 \\
\hline
\end{tabular}

Table 7. As in table 6 but to $\mathcal{O}\left(\frac{v^{2}}{f^{2}}\right) \cdot x_{H}=\frac{5 t_{W}}{4\left(5-t_{W}^{2}\right)}$ is defined in eq. (2.32) with $t_{W}=\frac{s_{W}}{c_{W}}$.

Open Access. This article is distributed under the terms of the Creative Commons Attribution License (CC-BY 4.0), which permits any use, distribution and reproduction in any medium, provided the original author(s) and source are credited. 